\title{
Design procedure of first-order perturbations for collisions trajectories with a perturbing body
}

\author{
Vera Petelina* \\ Moscow State University of Civil Engineering, 129337, Moscow, Russian Federation
}

\begin{abstract}
The article is devoted to the determination of first- and secondorder perturbations in rectangular coordinates and velocity components of body motion. Special differential equation system of perturbed motion is constructed. The right-hand sides of this system are finitesimal polynomials in powers of an independent regularizing variate. This allows constructing a single algorithm to determine first- and second-order perturbations in the form of finitesimal polynomials in powers of regularizing variates that are chosen at each approximation step. Following the calculations results with the developed method use, the coefficients of approximating polynomials representing rectangular coordinates and components of the regularized body speed were obtained. Comparison with the numerical results of the disturbed motion equations shows their close agreement. The developed method make it possible to calculate any visa point of body motion by the approximating polynomials.
\end{abstract}

\section{Introduction}

One of the crucial tasks associated with trajectory measurements is the determination of the partial derivatives of rectangular coordinates that make up the body motion speed with respect to the initial conditions. Auxiliary functions, which are degree series with respect to the additional variable, were introduced in the article [1]. In addition, operating procedures of universal variables in a number of mechanics problems to determine disturbances by the constants variation method were presented in the article [2]. Whereby, initial values components of the radius-vector and velocity were considered as osculating variables. New methods of disturbances determination keep standard features of classical ones, the small parameter method is used while disturbances calculation, which makes it possible to obtain solution asymptotic decomposition. Recently, Picard method for integration of differential equations is more commonly used, which leads to a convergent process of successive approximations that gives a differential equations system solution.

The error of solution depends on the initial approximation accuracy of the perturbation function. General development principles of perturbation theory in coordinates were studied the article [2] as the use of regularizing variables for path-planning calculation. Research

\footnotetext{
* Corresponding author: verapetelina51@gmail.com
} 
reveals that the regularizing variables use increases the calculations accuracy and significantly reduces the calculation time.

A crucial task of mechanics is to approximate the rectangular coordinates that make up the body speed and time in case of disturbed motion by algebraic polynomials of the lowest degree with respect to the auxiliary variable with a predetermined degree of accuracy.

One of the important problems in mechanics is the approximation of rectangular coordinates, the regularizing velocity constituents and the body motion time by the algebraic polynomials in power of the lowest degree additional variable with a prescribed degree of accuracy.

This article describes a differential equation system of the perturbed body motion. This system is ntegrated through successive approximations method, which gives insight into rectangular coordinate and body motion constituents in the form of polynomials in powers of some specially introduced additional variable that appears at each approximation step.

\section{Mathematical model}

Equations that integrate rectangular coordinates, constituents of regularized velocity and time with additional variables $C_{i}$ were found in works [3-5] as follows:

$$
\begin{gathered}
x-x_{0}+\sum_{i-1}^{7} C_{i} \cdot q_{1}^{(i)} x^{\prime}-x_{0}{ }^{\prime}+\sum_{i-1}^{7} C_{i} \cdot q_{4}^{(i)} \\
y-y_{0}+\sum_{i-1}^{7} C_{i} \cdot q_{2}^{(i)} y^{\prime}-y_{0}^{\prime}+\sum_{i-1}^{7} C_{i} \cdot q_{5}^{(i)} \\
z=z_{0}+\sum_{i-1}^{7} C_{i} \cdot q_{3}^{(i)} z^{\prime}-z_{0}^{\prime}+\sum_{i-1}^{7} C_{i} \cdot q_{6}^{(i)} \\
t=t_{0}-\sum_{i-1}^{7} C_{i} \cdot q_{7}^{(i)}
\end{gathered}
$$

where: - $\quad q_{i}^{(k)}(i, k-1, \ldots, 7)$ is partial solutions of equations in variations of regularized equations of a problem with 2 bodies. In order to find partial solutions it $q_{i}^{(k)}(i, k=1, \ldots, 7)$ is necessary to differentiate the general solution of regularized equations

of an unperturbed problem with 2 bodies, by initial data $\xi_{0}, \eta_{0}, \zeta_{0}, \xi_{0}{ }^{\prime}, \eta_{0}{ }^{\prime}, \zeta_{0}{ }^{\prime}, t_{00}$.

In work [5] the following equations for defining first-degree perturbations in values $C_{i}$ were found:

$$
C_{i}=\frac{\mu_{1}}{\mu} \cdot \frac{p_{0}^{2}}{a_{1}^{2}} \cdot \int_{-1}^{u}\left[\frac{a_{1}^{3}}{\Delta_{1}^{3}} \cdot\left(E_{i x}^{*} \cdot \frac{x_{1}-x_{0}}{a_{1}}-E_{i y}^{*} \cdot \frac{y_{1}-y_{0}}{a_{1}}\right)+\frac{a_{1}^{3}}{r_{1}^{3}} \cdot\left(-E_{i x}^{*} \cdot \frac{x_{1}}{a_{1}}-E_{i y}^{*} \cdot \frac{y_{1}}{a_{1}}\right)\right] \cdot \frac{d 3}{d u} \cdot d u
$$

$(i=1,2,4,5,7)$ 


$$
C_{i}-\frac{\mu_{1}}{\mu} \cdot \frac{\rho_{0}^{2}}{a_{1}^{2}} \cdot \int_{-1}^{u}\left[\frac{a_{1}^{3}}{\Delta_{1}^{3}} \cdot E_{i z}^{*} \cdot \frac{z_{1}-z_{0}}{a_{1}}+\frac{a_{1}^{3}}{r_{1}^{3}} \cdot\left(-E_{i z}^{*}\right) \cdot \frac{z_{1}}{a_{1}}\right] \cdot \frac{d \beta}{d u} \cdot d u
$$

$(i=3,6)$

where $E_{i x}^{*}, E_{i y}^{*}, E_{i z}^{*}$ are additional variables which represent polynomials in powers of regularized variable $\beta$

$$
\begin{gathered}
E_{1 x}^{*}=\beta^{E_{y}^{*}}=\frac{\sqrt{2}}{3} \cdot \beta^{3} \\
E_{1 y}^{*}=-\sqrt{2} E_{5 x}^{*}-\sqrt{2} \cdot\left(-\beta-\frac{1}{2} \beta^{3}\right) \\
E_{2 x}^{*}=\sqrt{2} \cdot\left(1+\frac{3}{2} \beta^{2}\right) E_{5 y}^{*}-1-\frac{1}{4} \beta^{4} \\
E_{2 y}^{*}--3+\frac{1}{2} \beta^{3} E_{6 z}^{*}=1-\frac{1}{4} \beta^{4} \\
E_{3 z}^{*}=-\beta-\frac{1}{2} \beta^{3} E_{7 x}^{*}=\frac{1}{2} \beta^{2}+\frac{1}{3} \beta^{4}-\frac{1}{40} \beta^{6} \\
E_{4 x}^{*}=1+\beta^{2}-\frac{\beta^{4}}{12} E_{4 y}^{*}=\sqrt{2}\left(\frac{1}{6} \beta^{3}+\frac{3}{20} \beta^{5}\right)
\end{gathered}
$$

$x_{1}, y_{1}, z_{1}$ are the perturbing body coordinates, $x_{0}, y_{0}, z_{0}$ are the studied body coordinates in an unperturbed motion.

Thus, expression for values $C_{i}$ may be represented as follows

$$
C_{i}=\int_{-1}^{u} \frac{S_{i}(u)}{(1-u)^{2}} d u
$$

$(\mathrm{i}=1,2,4,5,7)$

Where

$$
\begin{gathered}
S_{i}(u)=\frac{\mu_{1}}{\mu} \cdot \frac{\rho_{0}^{2}}{a_{1}^{2}} \cdot\left[\frac{a_{1}^{3} \cdot G_{1}(u)}{1-u} \cdot\left(E_{i x}^{*} \cdot \frac{x_{1}-x_{0}}{a_{1}}+E_{i y}^{*} \cdot \frac{y_{1}-y_{0}}{a_{1}}\right)+\frac{a_{1}^{3}}{r_{1}^{3}} \cdot\left(-E_{i x}^{*} \cdot \frac{x_{1}}{a_{1}}-E_{i y}^{*} \cdot \frac{y_{1}}{a_{1}}\right)\right] \cdot \frac{d \beta}{d u} \\
\frac{d \beta}{d u}=\frac{1}{2} \beta_{1} .
\end{gathered}
$$

Functions $E_{i x}^{*}, E_{i y}^{*}$ may be represented with the help of as $3-\frac{1}{2} \cdot 3_{1} \cdot(1+u)$,

polynomials in powers of standardized regularizing variable $u$, whereas $\mathrm{t}$

$$
u=1, \beta=\beta_{1}, \beta_{1}=\sqrt{2 \cdot \frac{r}{\rho_{0}}-1} \text { Value }
$$


$\beta_{1}$ corresponds to the collision moment of the studied body with the perturbing body. Then, functions $S_{i}(u)$ are shown as polynomials in powers of $u$ as follows

$$
S_{i}(u)=\sum_{k=0}^{n_{5}} S_{i k} \cdot u^{k}+\varepsilon\left(S_{i}, n_{5}\right)
$$

and functions $C_{i}(u)$ fora hit trajectories into perturbing body look as follows

$$
C_{i}(u)=\int_{-1}^{u} \frac{S_{i 0}}{(u-1)^{2}} d u+\int_{-1}^{u} \frac{S_{i 1} \cdot u}{(u-1)^{2}} d u+\ldots+\int_{-1}^{u} \frac{S_{i k} \cdot u^{k}}{(u-1)^{2}} d u+\ldots
$$

$(i=1,2,4,5,7)$

Let us enter a new regularizing variable:

$$
w=1-u
$$

Then, expressions (8) will have the following form:

$$
C_{i}(1-w)=-\int_{2}^{w} \frac{S_{i 0} \cdot d w}{w^{2}}-\int_{2}^{w} \frac{S_{i 1} \cdot(1-w) \cdot d w}{w^{2}}-\cdots-\int_{2}^{w} \frac{S_{i k} \cdot(1-w)^{k} \cdot d w}{w^{2}}-\cdots
$$

Integrals are easy to calculate:

$$
\begin{gathered}
\int_{2}^{w} \frac{S_{i 0} \cdot d w}{w^{2}}=S_{i 0} \cdot\left(\frac{1}{w} \quad \frac{1}{2}\right) \\
\int_{2}^{w} \frac{S_{i 1} \cdot(1 \quad w) \cdot d w}{w^{2}}=S_{i 1} \cdot\left(\frac{1}{w} \mid \ln w \quad \frac{1}{2} \quad \ln 2\right) \\
\int_{2}^{w} \frac{S_{i 2} \cdot(1 \quad w)^{2} \cdot d w}{w^{2}}=S_{i 2} \cdot\left(\frac{1}{w} \mid 2 \ln w \quad w+\frac{3}{2} \quad 2 \ln 2\right) \\
-\int_{2}^{w} \frac{S_{i 3} \cdot(1-w)^{3} \cdot d w}{w^{2}}=S_{i 3} \cdot\left(\frac{1}{w}-3 \ln w-3 w+\frac{1}{2} w^{2}+\frac{7}{2}-3 \ln 2\right) \\
-\int_{2}^{w} \frac{S_{i 4} \cdot(1-w)^{4} d w}{w^{2}}=S_{i 4} \cdot\left(\frac{1}{w}+4 \ln w-6 w+2 w^{2}-\frac{1}{3} w^{3}+\frac{37}{6}-4 \ln 2\right) \\
-\int_{2}^{w} \frac{S_{i 5} \cdot(1-w)^{5} d w}{w^{2}}=S_{i 5} \cdot\left(\frac{1}{w}+5 \ln w-10 w+5 w^{2}-\frac{5}{3} w^{3}+\frac{w^{4}}{4}-\frac{53}{6}-5 \ln 2\right) \\
C_{i}(1-w)=\tilde{C}_{i}(w),
\end{gathered}
$$

$(\mathrm{i}=1,2,4,5,7)$

Thus, first-degree perturbations in additional variables $\dot{C}_{i}$ have the form:

$$
\tilde{C}_{i}(w)-\frac{P_{i 0}}{w}-P_{i 1} \cdot \ln w-Q_{i}(w)+\varepsilon\left(Q_{i}\right),
$$

where: 


$$
Q_{i}(w)=\sum_{k-0}^{n_{6}} Q_{i k} \cdot w^{k}+\varepsilon\left(Q_{i}, n_{6}\right)
$$

$(i=1,2,4,5,7)$

Particular equations solutions in regularized equations variations of a problem with 2 bodies, are shown in work [5] as polynomials in powers of regularizing variable $\beta$.By putting expression $\beta=\frac{1}{2} \cdot \beta_{1} \cdot(1+u)$, into expressions $q_{i}^{(k)}(\beta)$, we obtain particular solutions $q_{i}^{(k)}$ as polynomials in powers of regularizing variable $w$.

Unperturbed motion polynomials $x_{0}, y_{0}, x_{0}^{\prime}, y_{0}^{\prime}, t_{0}$ in powers of variable are deduced by a similar substitution. By putting the obtained expressions into formulas (1) we receive rectangular coordinates $x, y$, of regularized motion constituents $x^{\prime}, y^{\prime}$, time $t$ in the first approximation as follows

$$
\begin{gathered}
x-\frac{A_{1}}{w}-R_{x}(w) \cdot \ln w+R_{x 1}(w)+\varepsilon\left(R_{x 1}, n^{*}\right) \\
y=\frac{A_{2}}{w} \mid R_{y}(w) \cdot \ln w+R_{y 1}(w)+\varepsilon\left(R_{y 1}, n^{*}\right) \\
x^{\prime}-\frac{A_{3}}{w}+R_{x^{\prime}}(w) \cdot \ln w+R_{x^{\prime} 1}(w)+\varepsilon\left(R_{x^{\prime} 1}, n^{*}\right) \\
y^{\prime}=\frac{A_{4}}{w}+R_{y^{\prime}}(w) \cdot \ln w-R_{y^{\prime} 1}(w)+\varepsilon\left(R_{y^{\prime} 1}, n^{*}\right) \\
t=\frac{A_{5}}{w}-R_{t}(w) \cdot \ln w+R_{t 1}(w)+\varepsilon\left(R_{t 1}, n^{*}\right),
\end{gathered}
$$

where $R_{x}, R_{x 1}, R_{y}, R_{y 1}, R_{x^{\prime}}, R_{x^{\prime} 1}, R_{y^{\prime}}, R_{y^{\prime} 1}, R_{t}, R_{t 1}$ are polynomials in powers $w$, whereas, errors may be done arbitrary small at selection of a respective value $n^{*}$. In expressions (14) for rectangular coordinates, constituents of regularized velocity and time of the spacecraft perturbed velocity, there are available terms at $\frac{1}{w}$ and $\ln w \cdot$ Introduce a new regularizing variable $v_{1}$ as follows:

$$
\begin{aligned}
& \ln w=\alpha_{1} \cdot v_{1}+\beta_{1} \\
& w=e^{i_{1} v_{1}+\omega_{1}}=e^{3_{1}} \cdot\left(1-\frac{\alpha_{1} v_{1}}{1 !}+\frac{\alpha_{1}^{2} v_{1}^{2}}{2 !}+\cdots+\frac{\alpha_{1}^{n} v_{1}^{n}}{n !}+\cdots+\right) \\
& w^{-1}=e^{-l_{1} v_{1}-\dot{r}_{1}}=e^{-\bar{r}_{1}} \cdot\left(1-\frac{\alpha_{1} v_{1}}{1 !}-\frac{\alpha_{1}^{2} v_{1}^{2}}{2 !}-\frac{\alpha_{1}^{3} v_{1}^{3}}{3 !}+\cdots+\frac{(-1)^{n} \alpha_{1}^{n} v_{1}^{n}}{n !}+\cdots\right)
\end{aligned}
$$




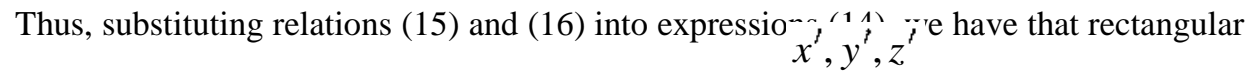
coordinates $x, y, z$, of regularized velocity constituents, time $t$ - are integral functions of a new regularizing variable $v_{1}$, which enables to present the specified functions by finitesimal polynomials with the prescribed accuracy degree.

Let us define values $\alpha_{1}$ and $\beta_{1}$ from boundary conditions that are $u=u^{*}, v_{1}=1$, at $u=-1, v_{1}=-1$. The value of regularizing variable $u^{*}$ or respectively $w^{*}$ correlates with the moment of the studied body getting into the effective area of the perturbing body and $u^{*} \prec 1, w^{*} \prec 1$. We have

$$
\left\{\begin{array}{l}
1-u^{*}=e^{i_{1}+i_{1}} \\
2=e^{i_{1}-\ddot{i}_{1}}
\end{array}\right.
$$

Where as $\alpha_{1} \prec \beta_{1}$

Thus by replacing a number of the independent first-degree perturbation variable in rectangular coordinates, regularized velocity constituents and bodies motion time, are represented as finitesimal polynomials of comparatively low degree and relatively regularizing variable with a sufficiently high accuracy degree.

Operating analysis diagram of first-order perturbations by analytical method consists of the following main blocks. The first block is concluded in building-up polynomials which show the perturbing body coordinates, coordinates, regularized velocity constituents and of unperturbed motion time of the studied body in powers of standardized regularizing variable $u$. Calculation results demonstrate respective polynomials coefficients.

The second block consists in representing the mutual distance square between the studied body and the perturbing body $\Delta_{1}^{2}$ as polynomial in powers of independent regularizing variable $u$.

In the third program block equation roots are found $\Delta_{1}^{2}=0$ is carried out with the help of iterations, i.e. determination of those values of the independent regularizing variable $u$, at which the studied body collision with the perturbing body takes place and that we will call critical points. Numerical analysis of the closest pair of critical points near the main measurement interval of regularizing variable $u$ is carried out. It showed that point $u-1$ is such critical point for the hit trajectories into perturbing body at which the distance from the studied body to the perturbing body turns to zero, the remaining pairs of critical points which are complex-conjugate are at significant distances. Polynomials are built-up:

$$
F_{1}(u)=\frac{\Delta_{1}(u)}{(u-1)^{2}} \quad G_{1}(u)=\frac{1}{F_{1}(u)^{3 / 2}}
$$

In powers of regularized variable $u$. Results represent respective polynomials coefficients.

The fourth program block consists in identifying the main part of perturbations in additional variables $C_{i}$ in the expansion form in functions $S_{i}(u)$ 


$$
\begin{gathered}
C_{i}(u)=\int_{1}^{u} \frac{S_{i k} \cdot u^{k} \cdot d u}{(u-1)^{2}} \\
C_{\text {глав. }}=\frac{\mu_{1} \cdot p_{0}}{\mu} \cdot \int_{1}^{u} \frac{a_{1} \cdot G_{1}(u)}{1-u} \cdot\left(E_{i x}^{*} \cdot \frac{x_{1}-x_{0}}{a_{1}}+E_{i y}^{*} \cdot \frac{y_{1}-y_{0}}{a_{1}}\right) \cdot \frac{d 3}{d u} \cdot d u,
\end{gathered}
$$

$(i=1,2,4,5,7)$

Auxiliary functions determination $E_{i x}^{*}$ and $E_{i y}^{*}$ is carried out in the form of polynomials in powers of regularized variable $u$ Functions $E_{i x}^{*}$ and $E_{i y}^{*}$ are dimensionless factors with respect to $D_{i k} / D$, where $D$ - is a fundamental matrix determinant of equation partial solutions in variations of regularized equations of a problem with 2 bodies [5], $D_{i k}$ - respective algebraic complements of the partial solution matrix. New regularizing variable $w$ introduction and the main part determination of perturbations in additional variables $C_{i}$ in polynomial-transcendent form with respect to regularizing variable $w$ are performed. Fifth program block represents the indirect part determination of perturbations in additional variables $C_{i}$

$$
C_{\text {косв. } i}=\frac{\mu_{1} \cdot \rho_{0}^{2}}{\mu_{1}} \cdot \int_{-1}^{u} \frac{a_{1}}{r_{1}^{3}} \cdot\left(E_{i x}^{*} \cdot \frac{x_{1}}{a_{1}} \quad E_{i y}^{*} \frac{y_{1}}{a_{1}}\right) \cdot \frac{d \xi}{d u} \cdot d u,
$$

\section{$(i=1,2,4,5,7)$}

as polynomials in powers of regularizing variable $w$ and determination of general perturbations in additional variables $C_{i}$ in polynomial-transcendent form with respect to variable $w$.

The sixth program block consists in the determination of partial solutions of $q_{i}^{(k)}$ equations in variations of regularized equations of 2 bodies problem in the polynomials form in powers of regularized variable $w$ as well as in determination of rectangular coordinates, constituents of regularized velocity and time of perturbed motion of the studied body in the first approximation in polynomial-transcendent form with respect to independent regularizing variable $w$. Calculation results represent coefficients of respective polynomials.

The seventh program block consists in determination of rectangular coordinates, constituents of regularized velocity and time of perturbed motion of the studied body in the form of polynomials in powers of a new regularizing variable $v_{1}$, which is introduced, with the help of relation (15).

\section{Summary}

Thus, by replacing a number of independent first-degree perturbation variable in rectangular coordinates, constituents of regularized velocity and of bodies motion time are represented as finitesimal polynomials of comparatively low degree with respect to 
regularizing variable with a sufficiently high accuracy degree. At each of calculations phase, a reduction in degree of respective polynomials with the help of Chebyshev polynomials and the computation results control by the initial and final point were carried out. Additionally, normalizing of regularizing variable $w$ to reduce the degree of polynomials, which represent the required functions with the preset accuracy degree on interval $-1,1$ was conducted.

The suggested method may be used at simulating the deformation of metal-ridged outer shell plating of panels and interior shell plating made of aluminum foil. At developing the procedure for calculation of multi-layer construction structures which are subjected to the impact of shock-waves, as well in studying construction structures, in particular, crossbending of cantilevers, at simulating tensions and deformations in construction elements effected by static loads of various value and configuration. As well, the procedure may be used at calculation of the structure in terms of seismic effect.

\section{References}

1. W. Goodyear, A general method of variation of parameters for numerical integration Astron, 70, 524-526 (1965).

2. R. Broucke, Perturbations in rectangular coordinates by iteration, Celest.Mech, 1, 110-129 (1969).

3. V. Petelina, Revisiting the convergence of successive approximations in the problem of disturbed body motion, MATEC Web of Conferences, 117, 00135 (2017).

4. V. Petelina, Method for finding majorant function estimates required to determine Picard's successive approximations convergence domain, MATEC Web of Conferences, 196, 01016 (2018).

5. V. Petelina, About choosing the form of perturbed body motion differential equations system, IOP Conf. Series: Materials Science and Engineering, 456, 012123 (2018). 